\title{
La gubernamentalidad biopolítica: de la sociedad de control estatal al liberalismo*
}

Recibido: octubre 28 de 2017 | Aceptado: febrero 23 de 2018

DOI: 10.17230/co-herencia.15.29.3

\author{
Julia Urabayen** \\ jurabayen@unav.es \\ Jorge León Casero*** \\ jleon@unizar.es
}

Resumen El pensamiento de Foucault es una caja de herramientas que ha sido utilizada de muy diversos modos. Estas diferentes aplicaciones, sin embargo, no significan que su reflexión carezca de unidad. Para dar razón de esta afirmación este artículo realiza una reevaluación integral de la obra de Foucault centrada en la gubernamentalidad y muestra las articulaciones que dan razón de los principales poderes analizados por el pensador francés: soberano, disciplinario, biopolítico estatal y biopolítico (neo)liberal. El paso de unas tecnologías de poder a otras no implica que haya eras ni que los poderes desaparezcan. Lo que cambia es su prevalencia. El texto estudiará las diferencias del poder disciplinar y el biopoder, la transformación del poder disciplinario en biopoder estatal y la conversión de este en (neo)liberalismo.

\section{Palabras clave:}

Foucault, biopolítica, (neo)liberalismo, población, seguridad, homo economicus, vulnerabilidad social.

\section{Biopolitical governmentality: from a state-controlled society to liberalism}

Abstract Foucault's thought is a toolbox which has been used in a variety of ways. Those multiple uses, do not imply, however, that his philosophy lacks unity. In order to ground this assertion, this article performs a comprehensive re-assessment of the work of Foucault, focused on the notion of governmentality, and it discusses how the main powers analyzed by the French thinker are related: sovereign power, disciplinary power, state biopolitics, and (neo) liberal biopolitics. Passing from certain power technologies
* Este artículo recoge resultados de la investigación "Mapa de Riesgo Social" financiada por el Ministerio de Economía y Competitividad, Programa de I+D+i orientada a los Retos de la Sociedad (2013). Referencia: CSO2013. 42576-R.

* Doctora en Historia de la Filosofía. Profesora de la Universidad de Navarra, España. ORCID ID: 0000-00020409-6279

*** Profesor Ayudante, Universidad de Zaragoza, España. ORCID ID: 0000-0002-2939. 5531 
to others does not entail the existence of eras of power or the disappearance of powers. What changes is their prevalence. This study analyzes the differences between disciplinary power and biopower, the transformation of disciplinary power into state power and the transformation of this latter into (neo)liberalism.

\section{Keywords:}

Foucault, biopolitics, (neo)liberalism, population, security, homo economicus, social vulnerability.

Foucault utiliza a lo largo de sus trabajos dos métodos -el arqueológico y el genealógico- lo cual para algunos estudiosos de su pensamiento se ha convertido en un criterio de delimitación en dos etapas (Megill, 1979, pp. 451-503), a las que se uniría una tercera, que surgiría por un cambio en las temáticas. Ahora bien, la publicación de Dits et écrits y de los cursos del Collège de France ha modificado esa interpretación porque ha permitido ver que Foucault retoma viejas cuestiones y que muchos temas están presentes en las tres etapas (Nilsson \& Wallenstein, 2013, pp. 9-10; Foucault, 2009, p. 133). Las lecturas más comprehensivas han mostrado que su pensamiento, aunque ha sido utilizado en ámbitos muy diversos, ${ }^{1}$ posee un orden que ofrece claves muy importantes a la hora de valorar los diferentes poderes analizados (Veyne, 2008, pp. 197-198).

Siguiendo la línea de estas interpretaciones filosóficas, creemos que la delimitación de períodos en el pensamiento foucaultiano no es ni clara ni nítida (Fontana \& Bertani, 1997, p. 248). No solo los temas abordados con ambos métodos son los mismos, sino que el pretendido giro ético de este autor es, en el fondo, una articulación de las temáticas políticas (Terrel, 2010, pp. 4, 8-9), un reajuste de su noción de biopolítica que, al entroncar con el liberalismo, apela a la libertad, la individualidad y las tecnologías del yo, ${ }^{2}$ nociones del tercer

1 La bibliografía dedicada al filósofo francés es inabordable y su impacto en las ciencias sociales enorme. Por citar solo algunos de los trabajos más recientes: Bert \& Lamy (2014), Gallego Franco y Val Valdivieso (2013), Crampton \& Elden (2007); la bibliografía más extensa está dedicada a la biopolítica, el liberalismo y la gubernamentalidad: Burchell, Gordon \& Miller (1991), Nilsson \& Wallenstein (2013), Donzelot (1998, 2007), y los números 6 y 18 de la revista Foucault Studies, que incluyen artículos de Tellmann (2009), Read (2009), Hamann (2009), Binkley (2009), Brady (2014), Murray (2014), Lippert (2014), Mitchell \& Lizotte (2014) y Fridman (2014).

2 Foucault delimita así el liberalismo: "1) Aceptación del principio de que en alguna parte debe haber una limitación del gobierno, y que no sea simplemente un derecho externo. 2) El liberalismo es también una práctica: ¿dónde encontrar exactamente el principio de 
Foucault que, según nuestra opinión, son aspectos que no rompen con sus tesis centrales previas: el poder crea la realidad, individual y social, por medio de dispositivos que se entrecruzan y superponen a lo largo de la historia. Lo que varía es la preponderancia de unos dispositivos sobre otros y con ello el sentido global de cada época histórica. Por ello, en su estudio de las tecnologías del yo, Foucault nunca afirma que exista un sujeto soberano fundacional ni universal (Foucault, 2009, pp. 136, 146). Ni tampoco asume que ese arte de existir sea una forma de conducir la vida al margen de las redes de los poderes-saberes.

En este trabajo nos centraremos en un momento fundamental del pensamiento foucaultiano con el objetivo de mostrar cómo se entretejen algunas de las cuestiones presentes desde antes, aunque más acentuadas a partir de la segunda mitad de la década de los setenta (Foucault, 2009, p. 62). Si se leen con detenimiento sus cursos de 1976 a 1979 se percibe que la atención del pensador francés se dirige hacia la biopolítica e igualmente se advierte que en el estudio de esas prácticas se cruzan dos reflexiones que parecen cortocircuitar el proyecto inicial: el liberalismo como forma política que pretende gobernar lo menos posible, y el poder pastoral vinculado al gobierno de sí y de los otros, más que al gobierno de los vivientes (Cubides, 2006, p. 79).

Este es un punto de confluencia de desarrollos previos que van cambiando respecto a lo proyectado -recuérdese que La historia de la sexualidad no solo quedó inacaba, sino que es un texto que tuvo transformaciones muy importantes (Castro, 2013, pp. 16-17)- pues cuando el francés analiza el gobierno de los individuos según la biopolítica señala que obedece a una multiplicidad de prácticas: el gobierno de sí, el gobierno de las almas y las conductas, el gobierno de los niños y el gobierno de los Estados (Foucault, 2006, pp. 110-111).

limitación del gobierno y cómo calcular los efectos de esa limitación? 3) El liberalismo es, en un sentido más restringido, la solución consistente en limitar al máximo las formas y los ámbitos de acción del gobierno. 4) Para terminar, el liberalismo es la organización de los métodos de transacción aptos para definir la limitación de las prácticas de gobierno: a) constitución, parlamento; b) opinión, prensa; c) comisiones, investigaciones" (Foucault, 2007a, p. 39). Además del liberalismo del siglo XVIII, Foucault analiza el ordoliberalismo y el neoliberalismo estadounidense que tienen en común el rechazo de la economía dirigida o del intervencionismo estatal (Foucault, 2007a, pp. 97-98). 
Estos son modos de poder que tienen en común ser artes de gobierno o gubernamentalidad, la noción que paulatinamente ganará más peso en su obra tardía. Al reflexionar sobre estas artes, Foucault destaca que hay una continuidad ascendente -para gobernar bien el Estado primero hay que gobernarse bien a sí mismo, a la familia y a sus propiedades, pues solo se puede ser dueño de los otros si se es dueño de sí mismo (2014, pp. 266-267)- y otra descendente (ese papel le corresponde a la policía), y sostiene que la pieza central del gobierno es la economía, pues se gobierna un complejo formado por los hombres y las cosas, lo que explica tanto el biopoder estatal como el biopoder liberal. ${ }^{3}$

Es la toma en consideración de estas diferentes artes de gobierno la que introduce los cambios en el proyecto foucaultiano. En función de qué arte de gobernar se estudie más, se estará en una visión más política o en otra más ética, la cual sigue siendo política, pues:

la gobernabilidad implica una relación del yo consigo, lo que significa exactamente que en la relación de gobernabilidad se está apuntando a la totalidad de las prácticas por las que se puede construir, definir, organizar e instrumentar las estrategias que los individuos, en su libertad, pueden utilizar con respecto a cada uno. Son los individuos libres quienes intentan controlar, determinar y delimitar la libertad ajena, y para hacerlo disponen de ciertos instrumentos para gobernar a otros. (Foucault, 2009, pp. 167-168)

La articulación de las prácticas de poder es, por tanto, compleja y requiere adoptar una visión comprehensiva de toda la obra

3 La economía es, a diferencia del derecho, una limitación interna de la política que se presenta como un medio adecuado para lograr los objetivos buscados por la acción gubernamental porque delimita esta acción haciendo que se atenga a las leyes naturales que regulan la producción e intercambio de bienes. Esto sucede alrededor de la mitad del siglo XVIII y se denomina economía política, término que denota el análisis de la producción y circulación de las riquezas, el método de gobierno que asegura la prosperidad de una nación así como una reflexión sobre la organización, distribución y limitación de los poderes en una sociedad. En los tres sentidos, la economía política surge en el marco de la razón de Estado y busca su enriquecimiento, lo que implicará que sus acciones deben ser juzgadas en función del éxito o el fracaso de sus efectos, no en función de la legitimidad. La relación entre estos factores es tan estrecha que el pensador francés identifica el naturalismo gubernamental con el liberalismo. Para Foucault el aspecto fundamental del nuevo arte de gobernar que surge a partir de allí consiste en que limita desde dentro el ejercicio del poder de gobernar, lo cual define al liberalismo (Foucault, 2007a, pp. 27-38, 79). 
foucaultiana. Los mismos temas aparecen en textos de los tres períodos y, lo que es más importante, Foucault no establece un corte entre eras de poder, ya que "las tecnologías del poder pueden ser transferidas de un campo a otro en el transcurso de la historia" (2009, p. 44). Si bien está claro que en las obras arqueológicas se ocupa más de la relación del saber y el poder, en ellas aparecen análisis del poder disciplinario, de su relación con el poder soberano, y de las líneas de conexión con lo que posteriormente denominará "biopoder".

Así pues, según Foucault, lo que cambia es la preponderancia de las técnicas y su horizonte de sentido, lo que no implica que desaparezcan. Por ello Lemke, al estudiar la recepción de la gubernamentalidad en las ciencias sociales, sostiene que ese término está dotado de una gran ambigüedad que hace inviable la pretensión de usarlo como una meta-narrativa que englobaría todos los demás tipos de poder conduciendo la historia hacia una mayor racionalización, lo que supone un fuerte idealismo y una delimitación de eras históricas; postulados contrarios a lo que sostiene Foucault. Como los poderes se superponen, la noción de gubernamentalidad está cargada de ambivalencia, rupturas y saltos (Lemke, 2013, pp. 35-52).

No hay, por tanto, linealidad ni desaparición de poderes. El poder soberano perdura en la sociedad en la que vivimos actualmente, ${ }^{4}$ lo mismo que el disciplinario, aunque según los últimos escritos de Foucault, el mundo que surge en torno a los años $80 \mathrm{y}$, en el que en gran parte continuamos, supone la prevalencia de la biopolítica centrada en la seguridad, y nutrida-nutriendo la lógica del (neo) liberalismo..$^{5}$ Además, el paso del biopoder como poder estatalizado al biopoder como liberalismo articulado en torno a la libertad está unido al poder pastoral: al cuidado de sí y de los otros. En el estudio

4 Desde la perspectiva del operaísmo italiano, en las sociedades predominantemente biopolíticas el poder soberano perdura como "poder de mando" capitalista. Un poder de mando cuya justificación ya no puede ser la del organizador de la producción, dada la aparición del obrero social como proletariado inmaterial auto-organizado, una vez que se ha producido la subsunción real de la sociedad en el capital (Negri, 2003).

5 Se conserva incluso la lógica de la seguridad de la biopolítica vinculada al poder estatal. Piénsese, por ejemplo, en los dispositivos desplegados en Europa en la denominada crisis del ébola. 
de este gobierno de sí y de los otros, Foucault se propone "pasar del análisis de la norma a[l de] los ejercicios del poder, y pasar del análisis del ejercicio del poder a los procedimientos, digamos, de gubernamentalidad" (2013, p. 14).

Todo ello implica que la gubernamentalidad está unida al estudio de las experiencias de las diferentes formas por las cuales el individuo se constituye como sujeto, que es un proceso en el que juegan un papel clave el decir veraz (parrhesía), el gobierno de sí (2012a, p. 52) y las tecnologías del yo (2014, pp. 36-37). Es decir, estas transformaciones de la gubernamentalidad se articulan con y desde las temáticas éticas, lo que Foucault denominó "estética de la existencia" (2009, p. 59).

En suma, los mayores cambios en el pensamiento foucaultiano se producen entre 1977-1979, pero en ese movimiento hay una continuidad temática, que se puede concretar en varios puntos: i) la biopolítica, que, al final, se convierte en gubernamentalidad, y para algunos intérpretes se agota; ii) la sexualidad, que es el gozne entre el poder disciplinario y el biopolítico, proyecto que sufre cambios muy importantes; y iii) la parresía o decir verdadero que tiene un sentido político (Foucault, 2012a).

Este entramado de preguntas que se plasman en el modo en el que se imbrican en la biopolítica-fase final del poder disciplinario (lo que se corresponde con el control estatal), el poder pastoral y el liberalismo es el que dota al término "biopolítica" de una ambigüedad enorme, que no se resuelve estableciendo un corte entre un segundo y un tercer Foucault, sino reflexionando sobre el sentido de ese entrecruzamiento de poderes. ${ }^{6}$

La relevancia de esta complejidad de la red de poderes y la ambivalencia de los términos "biopolítica" y "gubernamentalidad" son, probablemente, los que han producido una amplia y diversa recepción de la obra foucaultiana, especialmente intensa en las ciencias sociales. ${ }^{7}$ El objetivo de nuestro artículo no es repasar la

6 Según Mills, aunque la biopolítica se ha convertido en una disciplina, no se ha llegado a clarificar lo que se entiende por vida. Para lograrlo propone una reconsideración de la obra de Agamben y Esposito apoyándose en Canguilhem y Foucault (Mills, 2013, pp. 73 89). Sigue la misma línea argumental el trabajo de Reid, que recupera el papel del error en la vida apoyándose en Nietszche (Reid, 2013, pp. 91-104).

7 Vila señala dos líneas principales de recepción. La primera se desarrolla a partir del 
ingente literatura dedicada a la recepción de Foucault en esos saberes, sino mostrar cómo se articulan las nociones de poder disciplinario y biopolítica, así como los dos sentidos del biopoder en su obra.

\section{Poder disciplinario y biopoder}

Antes de centrarnos en esa enorme madeja de problemas que es la biopolítica, conviene recordar la noción de poder de Foucault. El pensador francés se enfrenta a una concepción que ve el poder como una propiedad que alguien posee y que puede transferir o alienar por un acto jurídico del orden de la cesión o el contrato con el objetivo de constituir una soberanía política.

Esa noción de poder como propiedad delegable no es algo que forme parte del pasado, sino que continúa vigente en los actuales discursos jurídicos del Derecho Constitucional, donde la soberanía del pueblo, vía la mediación representativa del Parlamento, es traducida en competencias cuya atribución constitucional a los distintos poderes puede ser cedida completamente a instituciones supraestatales (Unión Europea, Tratados Internacionales) o delegada a los distintos órganos administrativos estatales, autonómicos o municipales, y cuya gestión puede ser concedida a distintas empresas privadas. $^{8}$

Frente a esta interpretación del poder, Foucault señala que este funciona en red dando lugar al individuo y a la sociedad. Desde esta

cuerpo. La segunda destaca más el carácter político, lo que ha dado lugar a la diferencia entre biopolítica y biopoder (Vila, 2014, pp. 82-83). Esta es la posición de Hardt y Negri: "El biopoder está situado por encima de la sociedad, trascendente, a título de autoridad soberana que impone su orden. En cambio, la biopolítica es inmanente a la sociedad y crea relaciones y formas sociales a través de las formas colaborativas de trabajo" (2004, p. 124).

8 Un caso de especial relevancia es la elaboración de Planes Estratégicos a nivel municipal. Utilizando la ciudad de Zaragoza como ejemplo ilustrativo, su primer Plan Estratégico fue desarrollado entre 1994 y 1998 por Ebrópolis, una coalición de entidades públicoprivadas formada en 1994 e integrada por Ibercaja, la Fundación Caja Inmaculada, la CEOE, la Cámara de Comercio, CEPYME, CCOO, UGT, el Ayuntamiento de Zaragoza, el Gobierno de Aragón, la Diputación Provincial de Zaragoza, la Universidad de Zaragoza, la Federación de Asociaciones de Barrios de Zaragoza, y la Unión Vecinal Cesaraugusta. Este es el marco con el que se desarrolló tanto el Plan Integral del Casco Histórico de Zaragoza de 1997 como el Plan de Ordenación Urbana de 2001. En un inicio el número de entidades implicadas ascendía a trece. Para la conformación del Segundo Plan Estratégico en 2006 y la Estrategia Ebrópolis 2020, el número de socios había ascendido a doscientos setenta, la mayoría empresas privadas (Sorando, 2014; ASSI, 2015, pp. 10-19). 
óptica, aborda el poder disciplinario, que aparece en los siglos XVIIXVIII, y es un dispositivo que convive en las sociedades modernas con el poder soberano, que se ejerce a través del derecho público. El disciplinario, en cambio, lo hace a través de la mecánica polimorfa de las disciplinas que se expresa por medio de la norma (Foucault, 1997, p. 34).

Por ello, a diferencia de las rearticulaciones jurídico-constitucionales del poder soberano, las propias del poder disciplinario fueron realizadas predominantemente en el marco del derecho administrativo de la mano de su re-conceptualización como normalización y racionalización de los procesos de producción en los que interviene directamente el Estado. De ahí que el derecho administrativo en varios Estados europeos, España entre ellos, tuviera su origen y primeros desarrollos en las escuelas de ingenieros y no en las facultades de Derecho. ${ }^{9}$

El poder disciplinario se diferencia del soberano también porque lo ejerce la propia sociedad a través del control del tiempo y el espacio de cada individuo (el panóptico aplicado a todo tipo de instituciones). Es, pues, un poder disperso que cala en el cuerpo del individuo y lo moldea a fondo; es decir, es un poder individualizante y corrector cuyo modelo es médico. Este dispositivo se conserva igualmente en las sociedades actuales, pero ya no es el poder quien las define.

El biopoder, por su parte, produce y garantiza la seguridad de la sociedad, lo que incide en las nociones de riesgo y peligrosidad, que son conceptos que se van perfilando en los dispositivos disciplinarios (Foucault, 2007b, p. 258). Este poder se ocupa de datos relacionados con la población y busca optimizar la vida de un determinado grupo (Foucault, 1997, pp. 216-219). ${ }^{10}$ Los medios de los que se sirve son los mecanismos de previsión y de estimación estadísticos, así como los mecanismos reguladores para mantener una homeostasis y garantizar la seguridad acerca de todo lo aleatorio.

9 Puede encontrarse una aproximación a la rearticulación del derecho administrativo desde sus orígenes dentro del dispositivo disciplinario hasta su nuevo papel como garante de la gestión del riesgo en Esteve (2012).

10 Es importante recordar la diferencia entre el soberano y el súbdito que es gobernado por medio de las leyes; y la relación de la población y el biopoder, que pretende regular los aspectos relacionados con la vida de esa población, lo que requerirá operar mediante acciones que no son legales y que afectan, entre otras, a las condiciones que mejoran o empeoran la salud, la seguridad y el crecimiento de la población. 
Foucault señala, a modo de ejemplo, los mecanismos reguladores que inducen las conductas de ahorro, especialmente las que están unidas a la vivienda y su compra, los sistemas de seguros médicos o de seguros para la jubilación, ${ }^{11}$ las reglas de higiene dirigidas a asegurar la longevidad, y las presiones que los poderes políticos pueden ejercer sobre la sexualidad y la procreación así como sobre la higiene familiar, los cuidados de los niños y su escolarización (1997, p. 224). ${ }^{12}$ En todo caso, el modelo de este poder es, como en el disciplinario, médico y su objetivo es la seguridad de la población.

Sus dispositivos y su modo de legitimación no son los del poder soberano, pues, según Foucault, esto es el nacimiento de una nueva forma de gubernamentalidad (2006, pp. 84-86). El gobierno es, por tanto, diferente de la soberanía y se define como:

una manera recta de disponer las cosas para conducirlas [...] a un "fin oportuno" [...]. Creo que tenemos aquí una ruptura importante: mientras el fin de la soberanía está en sí misma y ella extrae instrumentos de sí con la forma de la ley, en el fin del gobierno están las cosas que dirige; debe buscárselo en la perfección o la maximización o la intensificación de los procesos que dirige, estos instrumentos, en vez de ser leyes, serán tácticas diversas. (Foucault, 2006, pp. 122-126)

Tampoco sus dispositivos son los propios del poder disciplinario. El elemento común (la norma) es el que permite la circulación de lo disciplinario a lo regulador, pues ambos son poderes normalizadores (Foucault, 1997, p. 225). Pero los dispositivos de seguridad funcionan de un modo diferente a los disciplinarios: "No se trata de intervenir menos sobre la sociedad, sino de intervenir de otro modo: menos reglamentar los procesos sociales, que manejarlos como realidades naturales dadas" (Vila, 2010, p. 192). Es decir, el biopoder toma en consideración el hecho biológico de que el hombre constituye una especie y se despliega como un conjunto de mecanismos que interactúan con los jurídico-legales y los disciplinarios-correctivos.

En el curso 1977-1978, Foucault muestra el cambio en el significado de la noción de población que se "considerará como un conjunto

11 La relación entre el riesgo y la seguridad, así como la tecnología de la seguridad son estudiados por François Ewald (1991, pp. 197-210) y por Daniel Defert (1991, pp. 211-234). Desde una óptica no foucaultiana el riesgo es analizado por Ulrich Beck (2001, 2006).

12 Estos ámbitos son gestionados-controlados gracias a los trabajadores sociales. Se puede encontrar un desarrollo del estudio de la policía de las familias en Donzelot (1998). 
de procesos que es menester manejar en sus aspectos naturales y a partir de ellos" (2006, p. 93). Es, pues, un fenómeno de la naturaleza que no se puede cambiar con un decreto, sino modificando aspectos relacionados con ella y especialmente a través del deseo generando un interés, lo que supone una modificación muy importante en la organización de las técnicas del poder. La consolidación de esta noción con sus aspectos biológicos y los productivos-consumistas sigue un proceso que culmina en la noción de biopolítica. ${ }^{13}$

Ahora bien, es fundamental comprender que estos poderes no actúan de la misma manera ni afectan-constituyen al mismo sujeto (el individual y el colectivo), ya que estas diferencias son las que permiten que el poder disciplinario y el biopoder no se excluyan, sino que se articulen (Foucault, 1997, p. 223). Es más, desde los primeros textos del francés el poder disciplinario está unido a las temáticas de la biopolítica (Trombadori, 2010, pp. 148-150). Es decir, para Foucault la individualidad es una producción biopolítica orientada al control de la productividad. El individuo es una realidad fabricada por la tecnología específica del poder denominada "disciplina” y este individuo es, a su vez, el sujeto de la biopolítica cuando se aborda su cuerpo productivo en su conexión con la sociedad, bien como especie, bien como máquina productiva-desiderante.

\section{La transformación del poder disciplinario en biopoder estatal}

Asumiendo la dificultad, o la imposibilidad, de realizar un corte entre estas tecnologías de poder, en este apartado vamos a abordar la gubernamentalidad propia del biopoder, de ese poder que a diferencia del disciplinario, crea el cuerpo social; aquel poder que, a diferencia del soberano, se ejerce no por vía legal, sino de manera capilar en el plano de lo social. De ese poder que a partir de un momento concreto se vincula a la razón de Estado y, a partir de otro, se ejerce por medio de unas artes de gobierno que están unidas, por una parte, al gobierno

13 Foucault desarrolla más lo relacionado con el arraigo biológico. La consideración de la creación del interés, de la publicidad y el consumo queda en su obra en un segundo plano. Estos son aspectos estudiados por Habermas (1981) en Historia y crítica de la opinión pública: la transformación estructural de la vida pública y por Marcuse (1983) en Eros y civilización. 
de sí y de los otros (poder pastoral) y, por otra, a una lógica política que nace con el liberalismo, inicialmente vinculada a la razón de Estado y posteriormente desarrollada como una "desinversión" en la que el Estado se desinteresa de ciertos aspectos relacionados con el orden interior (Foucault, 1978, pp. 5-7). La toma en consideración de todas estas articulaciones permitirá a Foucault cambiar radicalmente el sentido de biopolítica:

Este abordaje del liberalismo señala un giro en relación con su obra precedente. El desplazamiento teórico proviene de una autocrítica a su análisis de la biopolítica como unidimensional y reduccionista, en el sentido en que se centra principalmente en la vida biológica y física de una población y en las políticas del cuerpo. La introducción de la noción de gobierno ayuda a ampliar el horizonte teórico, ya que vincula el interés en una "anatomía política del cuerpo humano" con la investigación de los procesos de subjetivación y formas de existencia morales o políticos. (Lemm, 2010, p. 252) ) $^{14}$

El aspecto que hace las veces de hilo conductor en la transformación de sentido de la noción de biopolítica es el de seguridad. La biopolítica, que presenta varias dimensiones en los últimos cursos que impartió Foucault en el Colegio de Francia así como en conferencias dictadas en diferentes países de América, es una gubernamentalidad articulada en torno a la noción de seguridad. Este modelo, según Foucault, se va imponiendo al incidir, en primer lugar, en las cuestiones relacionadas con el espacio, ${ }^{15}$ aunque el poder soberano y el disciplinario están también relacionados con este. En el caso de la seguridad, el espacio está unido a la ciudad vista como un espacio de circulación y de producción necesaria para satisfacer

14 Oskala realiza una crítica a los supuestos básicos del liberalismo y enfatiza el carácter biopolítico, en el sentido fuerte, del liberalismo (2013, pp. 53-71).

15 Estos dispositivos liberales de seguridad tienen su reflejo en la concepción de las metrópolis del siglo XXI caracterizadas por una pluralidad administrativa (el área metropolitana de Los Ángeles está compuesta por más de ciento sesenta administraciones públicas -municipios- independientes) en abierta competencia fiscal como medio de atracción de poblaciones de renta media o alta (García Vázquez, 2004, pp. 99-119), lo que da lugar a la creación de guetos voluntarios e involuntarios, así como a "una redistribución geográfica en detrimento de las zonas menos aseguradas" (Vila, 2014, pp. 270-271). Frente a este progresivo deslizamiento del territorio hacia la población como paso de la lógica soberana y disciplinar a la lógica biopolítica del gobierno, Zibechi señala que el territorio es el elemento primordial de los movimientos sociales latinoamericanos, que son capaces de resistir las políticas neoliberales centradas en el control de poblaciones (2012, p. 138). 
las necesidades vitales: en el XVIII se produce el desenclave espacial, jurídico, administrativo y económico de la ciudad primando el papel del espacio de circulación (Foucault, 2006, pp. 28-40). ${ }^{16}$

La seguridad se vincula, en segundo lugar, al tratamiento de lo aleatorio, especialmente a la escasez. ${ }^{17}$ Foucault señala que lo aleatorio se analiza en series y se denomina medio, que es el soporte de la circulación; ${ }^{18}$ y adquiere una forma de normalización que es específica, ya que los dispositivos de seguridad son centrífugos y tienden a ampliarse: permiten el desarrollo de circuitos cada vez más amplios, son dispositivos que no regulan, sino que dejan hacer y, con ello, dejan espacio para la libertad porque lo que se busca es captar el punto en el que van a producirse las cosas (Foucault, 2006, pp. 66-69). Es decir, la seguridad y la libertad están profundamente unidas. Esto da lugar a una correlación entre la técnica de seguridad y la población: el fin no es aislar y curar (modelo disciplinario de la lepra), sino tomar la población como un conjunto sin discontinuidad.

El objetivo de los dispositivos de seguridad, que son los que se aplican a la población, es saber cuáles son los coeficientes de morbilidad probables para intentar reducir las situaciones más desviadas respecto

16 Las mutaciones gubernamentales que supone este cambio de concepción de la ciudad como delimitación espacial con autonomía jurídica propia de la Edad Media a la de espacio de circulación de mercancías y capitales fue tratada en 1977 por Virilio, quien sostuvo que una parte de los resultados obtenidos por su equipo de investigación fue teóricamente "usurpada" por el equipo de investigación que Foucault había creado para realizar el estudio arquitectónico de las cárceles que daría lugar a Vigilar y castigar (Virilio, 2006, p. 43).

17 Un aspecto interesante en el análisis del tratamiento de la escasez es la aparición de la noción de pueblo diferenciada de la de población (Foucault, 2006, pp. 63-65), así como la unión del pueblo, fracaso de la formación de la población, con las contra-conductas (Lemm, 2010, p. 321). Según Hardt y Negri, frente a la creación de la población por parte del biopoder, el antagonismo de clase que subyace a toda técnica de poder genera su propio concepto de multitud como forma constituyente de construcción del común (2004, p. 127). Además, la multitud se diferenciaría del concepto de "masas". Mientras que, por una parte, "las masas también son diferentes del pueblo, ya que no pueden ser reducidas a una unidad o a una identidad única" (Hardt y Negri, 2004, p. 16), por la otra, no son reconducibles al concepto de multitud dado que, al contrario que en estas últimas, "la esencia de las masas es la indiferenciación" (Hardt y Negri, 2004, p. 16). Esto supone una transformación de las tecnologías del poder y del sujeto político que se constituye a través del juego de los diferentes poderes.

18 Esta noción está presente en el modo en el que los urbanistas intentan reflejar y transformar el espacio urbano (Foucault, 2006, pp. 40-41). Este medio, como lugar de intervención del poder para modificar la especie humana, es uno de los elementos fundamentales de la introducción de los mecanismos de seguridad. 
a la curva normal por medio de la medicina preventiva (modelo de la inoculación de la viruela). Este dispositivo supone afrontar la enfermedad en términos de cálculo de probabilidades, lo que implica trabajar con la noción de caso, de riesgo, de peligro y de crisis. ${ }^{19}$ Como se ve, el tratamiento que Foucault realiza de la biopolítica, apelando a las disciplinas de la seguridad, se dirige directamente a los aspectos medioambientales y a los más propiamente físicos de la población. El biopoder es, pues, el poder que se aplica al hombre vivo, a la especie, y produce la población, que es gobernada de un modo diferente a como lo son los individuos.

Para el francés, la biopolítica se va estatalizando cada vez más hasta que en el siglo XIX el Estado se hace cargo de la vida. Es decir, Foucault señala un punto concreto en el que el gobierno de la vida-población reclama una estatalización e igualmente indica que esto se produce tardíamente porque requiere "órganos complejos de coordinación y de centralización" (Foucault, 1997, p. 222). El desarrollo de esta gubernamentalidad que apela al Estado se vincula a la estadística y a cambios importantes que suceden a lo largo de los siglos XVII y XVIII (Foucault, 2006, p. 133), lo que permitió la consolidación del arte de gobernar como economía política y la implementación de los dispositivos de seguridad.

Antes de ver cómo funcionan esos dispositivos es importante recordar que la formación de este poder está vinculada a varios aspectos, que son los que explican la ambigüedad de la biopolítica y los cambios que aparecen en el proyecto foucaultiano. Esta gubernamentalidad aparece tardíamente, no solo porque requiere coordinación y centralización, sino porque surge desde tres "sustratos" diferentes: la pastoral cristiana, una técnica diplomático-militar y la policía como arte de gobernar. La idea de que se gobierna a los hombres alcanza su elaboración más nítida en la pastoral cristiana, que entiende el poder como cuidado que atiende a omnes et singulati (Foucault, 2006, pp. 149-258). Foucault dice que la gubernamentalidad política se configura en el siglo XVI con los procedimientos propios del pastorado y también con la noción de sujeto formada por ese

19 Algunas de las técnicas de seguridad son reactivaciones y transformaciones de las técnicas jurídico-legales y de las disciplinarias. 
poder: "un sujeto atado a redes continuas de obediencia, un sujeto subjetivado por la extracción de verdad que se le impone" (2006, p. 219). ${ }^{20}$

El paso de ese poder pastoral a la gubernamentalidad ocurrió cuando el poder pastoral entró en crisis y se dispersó, lo que dio origen a contraconductas que buscaban otras formas por las cuales los sujetos fueran conducidos o simplemente la manera de conducirse cada uno a sí mismo (Foucault, 2006, pp. 223-225). La nueva concepción de la política, la estatal, nace de esas resistencias que erosionaron el poder pastoral. Como señala el francés, no se produjo una transferencia masiva de las funciones pastorales de la Iglesia al Estado, pero paulatinamente el biopoder fue adquiriendo nuevas tareas que se definen como propias del arte de gobernar (2006, pp. 275-276). Más concretamente, de ese arte de gobernar que atiende al individuo más que a la población en sentido medioambiental.

Esa es una modulación posterior del biopoder pues, según Foucault, el arte de gobernar es identificado inicialmente con la razón de Estado (2006, pp. 301-304). Desde esta perspectiva se ve que el arte de gobernar busca la pervivencia del gobierno y para ello acude a los remedios que evitan la sedición, lo que requiere equilibrar los recursos y la población aplicando toda una serie de técnicas que son las que el gobernante debe conocer: estadística o arcana imperii. ${ }^{21}$

En el gobierno de la población y en el fortalecimiento de la fuerza del Estado se introdujeron dos herramientas ya mencionadas: un dispositivo diplomático-militar y un dispositivo policial. Con el primero se buscó el equilibrio de Europa, lo que supuso un nuevo uso de la guerra, junto a una profesionalización de lo militar, y un desarrollo del instrumento diplomático (Foucault, 2006, pp. 341-353).

La policía, segundo mecanismo, cumplió una función clave: "el control y la cobertura de la actividad de los hombres" (Foucault, 2006, p. 369). Por ello se ocupó de la población: el número de hombres, los artículos de primera necesidad, la salud, el trabajo y la circulación. Es decir, siguió la misma lógica de atención a todos los

20 Los dos aspectos del pastorado que pasan a la biopolítica son su preocupación por lo humano y su carácter individualizador. Esto será muy relevante a la hora de entender el viraje de la biopolítica como control estatal hacia la biopolítica liberal que supone y consume libertad.

21 Estos temas son estudiados por Ian Hacking (1991, pp. 181-195). 
aspectos concretos propia del poder pastoral. Aquí el gobierno que ejerce el Estado, apelando a dispositivos de seguridad, y siguiendo una lógica biopolítica de protección-promoción de la población va virando hasta conceder más importancia a la libertad, que siempre está unida a la seguridad.

Para clarificar la diferencia en el modo en el que operan los dispositivos de seguridad en los dos tipos de biopolítica, conviene no olvidar que en el caso del control estatal la población se define principalmente desde el punto de vista biológico. La población es, como hemos señalado, diferente del sujeto colectivo creado por el contrato social y del pueblo sujeto a un poder soberano. Esta matización muestra su importancia al analizar la relación del biopoder con el discurso de las razas. Para Foucault uno de los discursos utilizados para explicar el poder como diferente de la soberanía es el que apela a la guerra, una de cuyas formulaciones es la guerra de las razas. ${ }^{22}$

Esta es una mecánica del poder que se funda sobre el cuerpo colectivo (no sobre la tierra ni sobre el cuerpo individual) y se constituye en torno a un tipo de disciplinas que buscan la normalización de lo biológico, lo que producirá un racismo biológico-social que establece una separación-lucha entre la raza verdadera y única, y la otra raza, la que no es autóctona ni sana, que deja de ser un enemigo externo para convertirse en aquello que se infiltra en el cuerpo social y se constituye en una amenaza para el patrimonio biológico (Foucault, 1997, pp. 47-53). De ahí la necesidad de seguridad: la población es vista como algo que hay que defender frente a los ataques "biológicos".23

Esta es la guerra que se libra en el cuerpo social o nación, pero se realiza no solo socialmente sino también estatalmente, pues una nación

22 La noción de nación no aparece por contrato, sino a partir del discurso de la guerra de razas y define a una sociedad como una asociación de individuos que comparte costumbres, usos y leyes comunes. La nación se constituye como el objeto del relato histórico y este proceso se articula en torno a tres ejes: filológico, económico-político y biológico. En esta línea de reflexión, Foucault muestra que el poder no está inicialmente centralizado en una institución (habitualmente identificada con el Estado) y señala que la lógica de la guerra de las razas, que se encuentra en el origen del biopoder, es social, no estatal (Foucault, 1997, pp. 75-76).

23 De ahí surgirá la idea de que el enemigo social es el desviado, el que no cumple la norma. Foucault dedica varias obras al estudio de los que no cumplen esa "normalidad". Entre otras, Les Anormaux (1999) y La vida de los hombres infames (1996). 
es más fuerte cuantas más capacidades estatales tiene. ${ }^{24}$ Así pues, este discurso que surge en la sociedad requiere para su puesta en práctica un poder centralizado y centralizador que adopta la forma de lucha por la vida y otorga al Estado la función de protector de la pureza solo cuando toma la forma de racismo de Estado y logra la estatalización de lo biológico. Ninguno de estos dispositivos es soberano.

\section{Del biopoder estatal al (neo)liberalismo}

A esta gubernamentalidad vinculada al Estado, sigue otra que es un tipo de arte de gobernar opuesto al anterior y que se apoya, a partir del siglo XVIII, en el papel de los economistas quienes "al recortar así el dominio del Estado [...] definieron una nueva racionalidad. [...] gubernamentalidad de los economistas que, creo, va a servir de introducción a algunas de las líneas fundamentales de la gubernamentalidad moderna y contemporánea" (Foucault, 2006, pp. 397-399). ${ }^{25}$

La idea clave, que servirá de paso al nuevo sentido de la biopolítica, es que el Estado tiene que garantizar la gestión de la población, lo que requiere un conocimiento científico porque la población tiene sus leyes propias de transformación y desplazamiento, y está sometida a procesos naturales (2006, pp. 402-403). Foucault cree que aquí se ha producido un cambio importante en la comprensión y el gobierno de la población, que ahora corresponde a quienes tienen los conocimientos científicos de la economía.

Además, hay que tener presente que en la fase precedente el modelo disciplinario ha ido cediendo su lugar a los mecanismos de seguridad: gobernar respetando las libertades. ${ }^{26}$ La nueva gubernamentalidad se articula, por tanto, con varios elementos: sociedad, economía, población, seguridad y libertad. Esto significa que esta gubernamentalidad establece una limitación interna que requiere para alcanzar sus objetivos limitar la acción gubernamental

24 Esto tiene lugar con la teoría del tercer Estado de Sièyes (Foucault, 1997, pp. 169-188).

25 Es aquí donde se da el nuevo sentido de la biopolítica, que para algunos intérpretes, como ya se ha señalado, no es compatible con el anterior.

26 Este cambio conduce al significado negativo que posee actualmente la policía y la nueva ideología jurídico-administrativa como gestión del riesgo (Pasquino, 1991, pp. 105-118). 
gracias al establecimiento de cosas que deben hacerse y cosas que no deben hacerse.

La cuestión es, por tanto, cómo no gobernar demasiado y la racionalidad que se utiliza para hacerlo es la economía política (Foucault, 2007a, p. 30), ${ }^{27}$ que reflexiona sobre las prácticas gubernamentales y las juzga por sus efectos, poniendo a la vez de relieve que hay una naturaleza propia de los objetos de la acción gubernamental, y que hay procesos y regularidades que se producen necesariamente debido a mecanismos inteligibles.

Así pues, la práctica gubernamental solo alcanzará su objetivo si respeta esa naturaleza, lo que implica conocerla. Esa autolimitación del gobierno es el liberalismo, que "es la organización de los métodos de transacción aptos para definir la limitación de las prácticas de gobierno [...] en lugar de tropezar con límites formalizados por jurisdicciones, se [da] a sí misma límites intrínsecos formulados en términos de veridicción" (Foucault, 2007a, p. 39). ${ }^{28}$

En este punto, el francés se centra en el liberalismo como autolimitación de la gubernamentalidad, más que en la población o la biopolítica. Foucault introduce un viraje en su proyecto y destaca que el liberalismo supone un dejar hacer en el ámbito de la economía y del mercado, que revela una verdad (la de los mecanismos naturales) que servirá para discernir las prácticas gubernamentales correctas de las erróneas. Y esta es una nueva forma de limitación del gobierno que se hizo fuerte y que, tomando como eje la utilidad, sirvió de criterio de establecimiento de los límites del poder público. ${ }^{29}$

Ello supuso una nueva definición del equilibrio europeo, que es visto como un sujeto económico que debe avanzar por el camino del progreso ilimitado, lo que conlleva un cálculo planetario que se limitará, no para respetar la libertad de los individuos, sino por la evidencia del análisis económico:

27 Esta primacía de la economía política que conlleva el paso del derecho a las ciencias naturales y sociales como técnica paradigmática del ejercicio del poder ya fue destacada por Marx en los Grundrisse (Marx, 1976, p. 540).

28 No hay ejercicio del poder sin aletúrgia. Este tema es estudiado en el curso 1979-1980 (Foucault, 2012b) y será clave en un gran número de los textos que para algunos autores forman parte del tercer Foucault, del que se ocuparía de la ética, el yo y el sujeto, más que de la política. En cambio, conviene destacar, por ejemplo, el papel político que el propio Foucault otorgó a la parresía.

29 El liberalismo se une a la fobia al Estado que apareció a finales del XVIII (Foucault, 2007a, pp. 92-98). 
Si empleo el término "liberal" es ante todo porque esta práctica gubernamental que comienza a establecerse no se conforma con respetar tal o cual libertad, garantizar tal o cual libertad. Más profundamente, es consumidora de libertad. [...] Consume libertad: es decir que está obligado a producirla. Está obligado a producirla y está obligado a organizarla. [...] en el corazón mismo de esa práctica liberal se instaura una relación problemática, siempre diferente, siempre móvil entre la producción de la libertad y aquello que, al producirla, amenaza con limitarla y destruirla. [...] Es preciso por un lado producir la libertad, pero ese mismo gesto implica que, por otro, se establezcan limitaciones, controles, coerciones, obligaciones apoyadas en amenazas, etcétera. (Foucault, 2007a, p. 84)

Conviene no perder de vista que esta apelación a la economía y a la libertad económica supone un rechazo del Estado como institución que controla e interviene. Por ello en esta lógica liberal la economía es la que produce la legitimidad del Estado y es la creadora de derecho público así como del consenso permanente de carácter político. ${ }^{30}$ Esto es, una razón gubernamental que busca legitimar un modo de relacionarse en el que la libertad económica se convierte en criterio político. De ahí que en este caso haya que pasar de la doctrina económica del liberalismo (que está presente en la consolidación de la razón de Estado y en la biopolítica como control estatal) al liberalismo como arte de gobernar.

Esta pretensión de que el mercado o la economía tiene capacidad de formalización para el Estado y para la sociedad es el primer elemento que modifica radicalmente el paso del liberalismo al neoliberalismo: "no se trata simplemente de liberar la economía. Se trata de saber hasta dónde podrán extenderse los poderes políticos y sociales de información de la economía de mercado" (Foucault, 2007a, p. 150). Por ello la política neoliberal no se ocupa del bienestar social, a lo sumo fomenta una 'política social individual' o privatizada; es decir, no se asegura a los individuos ante los riesgos con una cobertura social, sino que se otorga a cada uno de ellos un espacio económico dentro del cual cada uno asumirá y afrontará dichos riesgos. ${ }^{31}$

La gubernamentalidad neoliberal es, por tanto, un gobierno de la sociedad en el que esta es una sociedad de empresa (Foucault, 2007a, pp. 183-186). La sociedad es comprendida y regulada con los criterios

30 Rawls (1990).

31 Esta es la posición del anarcocapitalismo (Nozick, 1988). 
de la economía, la que, a su vez, tiene como principio fundamental la competencia: este es el segundo aspecto que el neoliberalismo modifica respecto al liberalismo clásico, pues concibe el mercado no como intercambio, sino como competencia. De ahí que la dinámica social, que se define en la economía, se identifique con la dinámica competitiva que es "un juego formal entre desigualdades" (Foucault, 2006, p. 153).

Como esto no es un dato primitivo, sino un constructo, la conclusión es clara: hay que gobernar para el mercado y pensar la gubernamentalidad desde esos principios del gobierno neoliberal que conciben la sociedad como una empresa, la población como capital humano y el hombre como empresario de sí mismo (Foucault, 2007a, pp. 255, 263-264). Es decir, el modelo de las relaciones sociales es económico, especialmente porque se ha producido una identificación del análisis económico con toda conducta racional, que es lo que permite juzgar la gubernamentalidad: sus intervenciones no se realizan sobre la economía, sino sobre la sociedad, pero el criterio de estas intervenciones es económico y se llevan a cabo porque hay "que intervenir sobre esa sociedad para que los mecanismos competitivos, a cada instante y en cada punto del espesor social, puedan cumplir el papel de reguladores" (2007a, p. 179). ${ }^{32}$

Este es el criterio de juicio con el que la gubernamentalidad biopolítica seguirá gobernando a los seres humanos, que ahora son capital humano, es decir, el resultado de inversiones y previsiones que cada cual realiza para garantizar el máximo posible de seguridad ante los riesgos y contingencias que puede padecer. En la idea de capital humano se diferenciará entre los elementos innatos ${ }^{33}$ y los adquiridos, que requieren inversiones educativas, que pueden calcularse. A este cálculo "se orientan las políticas culturales, las políticas educacionales de todos los países desarrollados" (Foucault, 2007a, p. 273)..$^{34}$

De ahí que la dinámica competitiva de la concepción empresarial de lo social fomente el ideal ético de la autosuperación y el esfuerzo,

32 Hasta la extrema pobreza se trata con la racionalidad neoliberal.

33 Foucault destaca la importancia que adquiere progresivamente la genética y su relación con la economía (Foucault, 2007a, pp. 268-269).

34 Bourdieu estudia estas temáticas educativas (Bourdieu, 2000, 2008; Bourdieu y Passeron, 1973) en contraposición a la anterior línea estructuralista desarrollada por Althusser (1996). 
y la división de la población en dos grupos: activos y en peligro de exclusión (Vila, 2014, p. 156). Los aspectos ético-sociales de esta visión del hombre empresario de sí y el establecimiento de dos grupos sociales desde el punto de vista económico están sujetos ahora a nuevas técnicas de gobierno, que priman la libertad, pero lo hacen al precio de culpar de su propio "fracaso" a quien no alcanza ciertos estándares (Donzelot, 1991, pp. 251-280).

Una muestra de este viraje en la gubernamentalidad es el gran peso estadístico concedido a los indicadores socio-económicos en el análisis de la vulnerabilidad urbana: 1) Porcentaje de parados respecto al total de población activa. 2) Porcentaje de la población de 16 a 29 años en situación de paro respecto al total de población activa de 16 a 29 años. 3) Porcentaje de ocupados que son trabajadores por cuenta ajena con carácter eventual o temporal sobre el total de ocupados. 4) Porcentaje de trabajadores no cualificados respecto al total de ocupados. 5) Porcentaje de población mayor de 16 años que no dispone de ninguna titulación académica (Ministerio de Fomento, 2012). Es decir, la gubernamentalidad neoliberal genera profundas desigualdades y divisiones sociales que ahora se considera que son el resultado del ejercicio de la libertad individual.

\section{Breves conclusiones}

Para Foucault, esta gubernamentalidad neoliberal crea y consume libertad y lo hace mediante un cálculo cuyo principio es la seguridad. Por ello la libertad es acompañada por técnicas disciplinarias de control, coacción y coerción. Es más, los dos conceptos forman parte de la misma lógica. ${ }^{35}$ Ello es así porque el neoliberalismo, a diferencia del liberalismo clásico, no se basa en el laissez-faire, sino en la vigilancia y control permanente de varios aspectos, entre los cuales se encuentran muchos que pueden ser englobados bajo la

35 Para Foucault no hay un salto entre la noción de libertad sin adjetivos y la libertad económica porque entiende el liberalismo, como ya se ha dicho, como una limitación del gobierno por la evidencia económica, lo que implica la libertad, que es definida como una relación entre gobernantes y gobernados en la que el gobierno produce lo que es necesario para que el gobernado sea libre, lo que supone necesariamente el establecimiento de límites y coacciones. Es decir, la libertad es algo que se fabrica. Y el principio del cálculo de la producción en este arte de gobernar es la seguridad de los individuos concretada en la noción de peligro: el panóptico y los dispositivos "liberógenos" (Foucault, 2007a, pp. $82-92)$. 
categoría de la política social. Esto significa que el neoliberalismo, además de una nueva manera de conducir la vida de los individuos o ethos, es una gubernamentalidad biopolítica.

Este dispositivo neoliberal no es una ruptura con la biopolítica, pero sí un cambio: ahora ya no es estatal, sino gubernamental. Así pues, el viraje de la gubernamentalidad biopolítica del control estatal al (neo)liberalismo no supone una modificación de las tecnologías de este tipo de gobierno, sino solo una modulación de ciertos dispositivos que, a fin de cuentas, desde el punto de vista social provocan un aumento de la vulnerabilidad. ${ }^{36}$

Por último, hay que considerar el sujeto que conforma, ya que el neoliberalismo, como todo dispositivo, produce un sujeto concreto que tiene una peculiaridad: "El homo oeconomicus es un hombre eminentemente gobernable" (Foucault, 2007a, p. 310).$^{37}$ A este individuo, económico y gobernable, le corresponde como correlato la sociedad civil, que es el conjunto en el que se resitúan esos sujetos para poder administrarlos convenientemente. ${ }^{38}$

Por lo tanto, lo que implica este cambio es una transformación en el tipo de sujeto que produce: el hombre autónomo, emprendedor que si "fracasa" queda excluido de lo social, no por ser anormal (poder disciplinario) ni peligroso (biopolítica estatalizada), sino por ser un marginado que no ha sabido gestionar bien sus recursos. El biopoder, incluso en su versión liberal, crea lo social y utiliza tecnologías que provocan desigualdades y disfunciones sociales, que pueden convertirse en tanatopolítica ${ }^{39}$

36 Foucault presenta la modificación de la gubernamentalidad biopolítica del EstadoProvidencia a la desinversión (neoliberalismo) que crea un nuevo orden interior sin una disciplina exhaustiva y destaca cuatro aspectos: la localización de zonas vulnerables en las que el Estado no desea que suceda nada, un aumento de los márgenes de la tolerancia que adquiere un carácter regulador, la aplicación de un dispositivo de poder que adopta la forma de sistema de información general, y la creación de un consenso por medio de los mass media que permite que el orden social se autorregule: son los sujetos económicos y sociales los que deben resolver los conflictos (Foucault, 1978, pp. 6-7).

37 Desde el punto de vista del homo economicus, Negri desarrolla su crítica al capitalismo y al socialismo como formas homólogas de producción y explotación de clase a través del trabajo (la primera basada en el derecho privado y la segunda en el derecho público) frente a una reconsideración del comunismo que, criticando abiertamente la ortodoxia de la Tercera Internacional y las lecturas economicistas del marxismo, vuelva a reconfigurarlo como abolición del trabajo (Negri, 2012, pp. 92, 100-101, 188).

38 Al seguir una lógica económica, es sobre y en la sociedad donde actúa y toma cuerpo este poder.

39 Para el francés el nazismo ha articulado el poder soberano y el biopoder en una 


\section{Referencias}

Althusser, L. (1996). Ideología y aparatos ideológicos del Estado. Buenos Aires: Nueva Visión.

ASSI-Acción Social Sindical Internacionalista (2015). Urbanismo neoliberal en Zaragoza. Planes de regeneración urbana y efectos socio-económicos en el barrio de San Pablo - El Gancho. Recuperado de https://bit.ly/2JHjKIv

Beck, U. (2001). La sociedad del riesgo. Hacia una nueva modernidad. Barcelona: Paidós.

Beck, U. (2006). La sociedad del riesgo global. Amok, violencia, guerra. Madrid: Siglo XXI.

Bert, J.-F. \& Lamy, J. (Eds.). (2014). Michel Foucault. Un héritage critique. Paris: CNRS Éd.

Binkley, S. (2009). The Work of Neoliberal Governmentality: Temporality and Ethical Substance in the Tale of Two Dads. Foucault Studies, 6, 60-78. doi: http://dx.doi.org/10.22439/fs.v0i0.2472

Bourdieu, P. y Passeron, J. C. (1973). Los estudiantes y la cultura. Barcelona: Labor.

Bourdieu, P. (2000). Capital cultural, escuela y espacio social. México: Siglo XXI.

Bourdieu, P. (2008). Homo academicus. Madrid: Siglo XXI.

Brady, M. (2014). Ethnographies of Neoliberal Governmentalities: from the neoliberal apparatus to neoliberalism and governmental assamblages. Foucault Studies, 18, 11-33. doi: http://dx.doi.org/10.22439/ fs.v0i18.4649

Burchell, G., Gordon, C., \& Miller, P. (Eds.). (1991). The Foucault effect. Studies in Governmentality. Chicago: University of Chicago Press.

Castro, E. (Ed.). (2013). Michel Foucault. La inquietud por la verdad. Escritos sobre la sexualidad y el sujeto. Barcelona: Siglo XXI.

Crampton, J. \& Elden S. (Eds.). (2007). Space, Knowledge and Power. Foucault and Geography. Hampshire: Ashgate.

Cubides, H. (2006). Foucault y el sujeto político. Ética del cuidado de sí. Bogotá: Universidad Central.

Defert, D. (1991). 'Popular Life' and Insurance Technology. In G. Burchell, C. Gordon \& P. Miller (Eds.), The Foucault effect. Studies in Governmentality (pp. 211-234). Chicago: University of Chicago Press.

tanatopolítica, pero esta unión está inscrita en el funcionamiento de todos los Estados (Foucault, 1997, p. 232). 
Donzelot, J. (1991). Pleasure in work. In G. Burchell, C. Gordon \& P. Miller (Eds.), The Foucault effect. Studies in Governmentality (pp. 251-280). Chicago: University of Chicago Press.

Donzelot, J. (1998). La policía de las familias. Valencia: Pre-Textos.

Donzelot, J. (2007). La invención de lo social. Ensayo sobre la declinación de las pasiones políticas. Buenos Aires: Nueva Visión.

Donzelot, J. (2008). Michel Foucault and liberal intelligence. Economy and Society, vol. 37, № 1, pp. 83-114. doi: https://doi. org/10.1080/03085140701760908

Esteve Pardo, J. (2012). Lecciones de Derecho Administrativo. Madrid: Marcial Pons.

Ewald, F. (1991). Insurance and Risk. In G. Burchell, C. Gordon \& P. Miller (Eds.), The Foucault effect. Studies in Governmentality (pp. 197210). Chicago: University of Chicago Press.

Fontana, A., \& Bertani, M. (1997). Situation du Cours. In M. Foucault, Il faut défendre la société: Cours au Collège de France 1975-1976 (pp. 247263; F. Ewald, M. Bertani \& A. Fontana, Eds.). Paris: Hautes Études (Gallimard - Seuil).

Foucault, M. (1978). Nuevo orden interior y control social. El viejo Topo, 7, 5-7. Recuperado de https://bit.ly/2ygpGmy

Foucault, M. (1996). La vida de los hombres infames. Buenos Aires: Museos de Buenos Aires.

Foucault, M. (1997). Il faut défendre la société. Cours au Collège de France (1976-1977). Paris: Gallimard.

Foucault, M. (1999). Les Anormaux. Cours au Collège de France (1974-1975). Paris: Seuil.

Foucault, M. (2005). Vigilar y castigar. Madrid: Siglo XXI.

Foucault, M. (2006). Seguridad, territorio y población. Curso en el Collège de France (1977-1878). Buenos Aires: Fondo de Cultura Económica de Argentina.

Foucault, M. (2007a). Nacimiento de la biopolítica: curso en el Collège de France (1978-1979). Buenos Aires: F. C. E.

Foucault, M. (2007b). El poder psiquiátrico. Curso en el Collège de France (1973-1974). México: F. C. E.

Foucault, M. (2009). El yo minimalista y otras conversaciones. Buenos Aires: La Marca.

Foucault, M. (2012a). Discurso y verdad en la antigua Grecia. Barcelona: Paidós. 
Foucault, M. (2012b). Du gouvernement des vivants. Cours au Collège de France (1979-1980). Paris: Gallimard.

Foucault, M. (2013). El gobierno de sí y de los otros. Curso (1982-1983). Madrid: Akal.

Foucault, M. (2014). Subjectivité et vérité. Cours au Collège de France. 19801981. Paris: Gallimard.

Fridmann, D. (2014). Resisting the lure of the paycheck: Freedom and dependence in financial self-help. Foucault Studies, 18, 90-112. doi: http://dx.doi.org/10.22439/fs.v0i18.4653

Gallego Franco, H. y Val Valdivieso, M. ${ }^{a}$ I. (Eds.). (2013). Las huellas de Foucault en la historiografía. Poderes, cuerpos y deseos. Barcelona: Icaria.

García Vázquez, C. (2004). La ciudad hojaldre. Visiones urbanas del siglo XXI. Barcelona: Gustavo Gili.

Habermas, J. (1981). Historia y crítica de la opinión pública: la transformación estructural de la vida pública. Barcelona: Gustavo Gili.

Hamann, T. H. (2009). Neoliberalism, Governmentality, and Ethics. Foucault Studies, 6, 37-59. doi: http://dx.doi.org/10.22439/fs.v0i0.2471

Hardt, M. y Negri, A. (2004). Multitud: Guerra y democracia en la era del Imperio, Barcelona: Debate.

Lemke, T. (2013). Foucault, Politics and Failure. In J. Nilsson \& S.-O. Wallenstein (Eds.), Foucault, Biopolitics and Governmentality (pp. 3552). Huddinge: Södertörn University.

Lemm, V. (Ed.). (2010). Michel Foucault: neoliberalismo y biopolítica. Santiago: Ediciones Universidad Diego Portales.

Lippert, R. (2014). Neo-liberalism, Police, and the Governance of Little Urban Things. Foucault Studies, 18, 49-65. doi: http://dx.doi. org/10.22439/fs.v0i18.4651

Marcuse, H. (1983). Eros y civilización. Madrid: Sarpe.

Marx, K. (1976). Elementos fundamentales para la crítica de la economía política (Grundrisse) 1857-1858. México: Siglo XXI.

Megill, A. (1979). Foucault, Structuralism, and the Ends of History. The Journal of Modern History, 51(3), 451-503. Recuperado de https://bit. ly/2tcyzaH

Mills, C. (2013). Biopolitical Life. In J. Nilsson \& S-O. Wallenstein (Eds), Foucault, Biopolitics and Governmentality (pp. 73-90). Huddinge: Södertörn University. 
Ministerio de Fomento, Gobierno de España (2012). Atlas de la Vulnerabilidad Urbana en España. Metodología, contenidos y créditos. Recuperado de https://bit.ly/22L89J3

Mitchell, K. \& Lizotte, C. (2014). The Grassroots and the Gift: Moral Authority, American Philanthropy, and Activism in Education. Foucault Studies, 18, 66-89. doi: http://dx.doi.org/10.22439/fs.v0i18.4652

Murray Li, T. (2014). Fixing Non-market Subjects: Governing Land and Population in the global South. Foucault Studies, 18, 34-48. Recuperado de https://bit.ly/2lcZRuh

Negri, A. (2003). La forma-Estado. Akal: Madrid.

Negri, A. (2012). Marx más allá de Marx. Cuaderno de trabajo sobre los Grundrisse. Madrid: Akal.

Nilsson, J. \& Wallenstein, S.-O. (Eds.). (2013). Foucault, Biopolitics and Governmentality. Huddinge: Södertörn University.

Nozick, R. (1988). Anarquía, Estado y utopía. México: F. C. E.

Oskala, J. (2013). Neoliberalism and Biopolitical Governmentality. In J. Nilsson \& S-O. Wallenstein (Eds), Foucault, Biopolitics and Governmentality (pp. 53-71). Huddinge: Södertörn University.

Pasquino, P. (1991). Theatrum Politicum: The Genealogy of Capital Police and the State of Prosperity. In G. Burchell, C. Gordon, \& P. Miller (Eds.), The Foucault effect. Studies in Governmentality (pp. 105118). Chicago: University of Chicago Press.

Rawls, J. (1990). Sobre las libertades. Barcelona: Paidós.

Read, J. (2009). A Genealogy of Homo-Economicus: Neoliberalism and the Production of Subjectivity. Foucault Studies, 6, 25-36. doi: http:// dx.doi.org/10.22439/fs.v0i0.2465

Reid, J. (2013). Towards an Affirmative Biopolitics. In J. Nilsson \& S-O. Wallenstein (Eds), Foucault, Biopolitics and Governmentality (pp. 91104). Huddinge: Södertörn University.

Sorando Ortín, D. (2014). Espacios en conflicto. Un análisis relacional del cambio social en los centros estigmatizados (tesis doctoral). Universidad Complutense de Madrid, España.

Tellmann, U. (2009). Foucault and the Invisible Economy. Foucault Studies, 6, 5-24. doi: http://dx.doi.org/10.22439/fs.v0i0.2487 
Terrel, J. (2010). Politiques de Foucault. Paris: PUF.

Trombadori, D. (2010). Conversaciones con Foucault. Pensamientos, obras, omisiones del último maître-à-penser. Buenos Aires: Amorrortu.

Veyne, P. (2008). Foucault. Sa pensé, sa personne. Paris: Albin-Michel.

Vila Viñas, D. (2014). La gobernabilidad más allá de Foucault. Un marco para la teoría social y política contemporáneas. Zaragoza: Prensas de la Universidad de Zaragoza.

Virilio, P. (2006). Speed and Politics. Los Angeles: Semiotext(e).

Zibechi, R. (2012). Territorios en resistencia. Cartografía política de las periferias urbanas latinoamericanas. Madrid: Baladre-Zambra, Ecologistas en Acción - CGT. 